complemented by a retrospective case-control, thematic analysis of electronic patient records, to examine the patient journey throughout the duration of the hospice stay.

Results LLOS admissions accounted for 23\%(76) of admissions to our hospice in 2015 (2342 bed days), median stay 28 days, 55\% female, median age 69 years, 96\% (73 admissions) referred for symptom control. Admission outcome: 38\% (29) of patients discharged, of which 59\% (17) had a new care arrangement following their stay.

No significant findings when analysing the following against LLOS admissions and admission outcome: Patient age, gender, primary diagnosis, number of comorbidities, presence of formal social care support, and permanent residence.

Thematic analysis identified 4 overarching themes implicated in LLOS: Uncertainty, In-house interventions, clinical and social complexity. This analysis emphasised, in particular, the interplay between communicating and managing uncertainty and social complexity.

Conclusion Our results would suggest that there are no sociodemographic or disease factors associated with LLOS. Thematic analysis provides an alternative and successful method of service evaluation. Thematic results emphasise the need for research into managing complexity and uncertainty in addition to highlighting the fact that our growing capacity to provide in-house interventions comes, ultimately, at a cost to bed availability.

\section{P-30 IMPROVING COMMUNICATION FROM ONCOLOGY TO PRIMARY CARE IN A LARGE CANCER CENTRE}

${ }^{1,2}$ Annette Edwards, ${ }^{1}$ Paul Hatfield, ${ }^{1}$ Fiona Hicks. 'Leeds Teaching Hospitals NHS Trust, Leeds, UK; ${ }^{2}$ Sue Ryder Wheatfields Hospice, Leeds, UK

\subsection{6/bmjspcare-2017-00133.30}

Background Recognising that people are entering the last phase of illness isn't always straightforward, and having conversations about treatment aims and planning for future care is not easy. In light of this a Senior Clinician Development Programme was established, comprising 7 consultants in different specialties and a GP to give a community perspective, facilitated by 2 palliative medicine consultants. This ran for 18 months from October 2011.

The following audit specifically looked at oncology, assessing if there was improvement in communication between secondary and primary care.

Method All letters to GPs of patients over 18 with a Leeds postcode previously under the care of an oncologist and who died in January $2010(n=79)$ and January 2014 $(n=82)$ were included. Patients whose treatment was curative and more than 10 years before their death were excluded if they died of an unrelated cause.

The improvement plan implemented between the two audits involved an oncology consultant participating in the development programme working alongside a GP in an Action Learning Set and spending time with District Nurses to understand their roles. He then worked with colleagues to highlight the importance of communication with GPs, patients and carers.

Results There was a statistically significant improvement in all desired characteristics of the letters between 2010 and 2014. This was most marked in recommendation to add the patient to the palliative care register. There was also a major improvement in documenting discussions with patients about the palliative nature of the disease and emphasis on quality of life.

Conclusion This audit shows that educational initiatives with system change can alter consultants' behaviour. This was a sustained effect, as funding for the development programme finished 2 years before re-auditing. Whilst other factors may contribute, it does demonstrate that long term change in consultant behaviour is possible, particularly when initiated by their peers with insight into department specific issues.

\section{P-31 A RETROSPECTIVE AUDIT OF SURVIVAL DURATION IN PATIENTS WITH POOR PERFORMANCE STATUS RECEIVING SYSTEMIC ANTI-CANCER THERAPY AT MID YORKSHIRE NHS TRUST}

1,2Emma Hooson, ${ }^{2}$ Charlotte Gibb, ${ }^{2}$ Gireesh Kumaran. 'St Gemma's Hospice, Leeds, UK; ${ }^{2}$ Pinderfields Hospital, Mid Yorkshire Hospitals NHS Trust, Wakefield, UK

\subsection{6/bmjspcare-2017-00133.31}

Background Research undertaken in patients with a cancer diagnosis and poor performance status (PS) has shown a lack of survival benefit or improved quality of life from chemotherapy. ${ }^{1,2,3}$ A review by the National Confidential Enquiry into Patient Outcome and Death found that poor PS was linked to an increased rate of death within 30 days of systemic anti-cancer therapy (SACT). ${ }^{4}$ We analysed survival duration in patients with a poor PS prescribed SACT in our local NHS trust.

Methods Details for all patients who were prescribed SACT in the Mid Yorkshire Hospitals NHS Trust from 1/1/2015 to 31/ $12 / 2015$ were obtained from the electronic prescribing system (chemocare) and electronic case records (PPM). Patients included had an Oncological ICD-10 classification and had a performance status of 2 or less at the start of cycle 1 of treatment. Duration of survival for these patients was calculated.

Results From the total 747 patients, 39 were identified to fit the criteria. 33 had a PS of 2, and 6 had PS of 3 at the first cycle of SACT. Common diagnoses were lung carcinoma $(n=23)$ and breast carcinoma $(n=8) .35$ patients received chemotherapy with palliative intent. 3 of these patients died within 30 days of receiving SACT, and a total of 8 patients died within 60 days (23\%). All 4 patients who received neoadjuvant, adjuvant or disease modifying chemotherapy were still alive at time of analysis, with a survival of at least 421 days.

Conclusions Nearly 1 in 4 patients with a PS of 2 or less who were prescribed palliative chemotherapy had died within 60 days of receiving SACT. Quality of life and best supportive care need to be the first consideration for patients with poor PS, but carefully chosen and counselled patients with chemosensitive disease can benefit from SACT (further research needed).

\section{REFERENCES}

1. Sánchez-Muñoz, Pérez-Ruiz, Sáez Ml, et al. Limited impact of palliative chemotherapy on survival in advanced solid tumours in patients with poor performance status. Clin Transl Oncol 2011;13(6):426-9.

2. Wright $A A$, Zhang $B$, Keating $N L$, et al. Associations between palliative chemotherapy and adult cancer patients' end of life care and place of death: prospective cohort study. BMJ 2014:4:348.

3. Prigerson $\mathrm{H}$, Bao $\mathrm{Y}$, Shah $\mathrm{M}$, et al. Chemotherapy Use, Performance Status, and Quality of Life at the End of Life. JAMA Oncol 2015:1 (6):778-784.

4. Mort D, Lansdown M, Smith N, et al. Systemic Anti-Cancer Therapy: For better for worse? National Conï $\neg$ dential Enquiry into Patient Outcome and Death [Online] Available at: http://www.ncepod.org.uk/2008report3/Downloads/ SAC T_report.pdf (accessed September 4 th, 2016) 